\title{
Diversity and distribution of vascular macrophytes in Ansupa Lake, Odisha, India
}

\author{
MUKTIPADA PANDA ${ }^{1,2, \boldsymbol{}}$, RABINDRO NATH SAMAL $^{1}$, KRUPA SINDHU BHATTA $^{1}$, SASMITA LENKA ${ }^{1}$, \\ JAJNASENI ROUT ${ }^{1}$, HEMANTA KUMAR PATRA $^{2}$, SUSANTA NANDA ${ }^{\mathbf{1}}$ \\ ${ }^{1}$ Chilika Development Authority, Department of Forest and Environment, Govt. of Odisha, Plot No. C-11, BJB Nagar, Bhubaneswar 751014, \\ Odisha, India. ^email: muktipadapanda@gmail.com \\ ${ }^{2}$ Post-Graduate Department of Botany, Utkal University. Bhubaneswar 751004, Odisha, India
}

Manuscript received: 14 March 2018. Revision accepted: 17 May 2018.

\begin{abstract}
Panda M, Samal RN, Bhatta KS, Lenka S, Rout J, Patra HK, Nanda S. 2018. Diversity and distribution of vascular macrophytes in Ansupa Lake, Odisha, India. Bonorowo Wetlands 8: 1-12. Macrophytes are an indispensable component of any wetlands. They are the base of the trophic structure and variously affect the function of the aquatic ecosystem. To identify the causative plant species, extensive invasion of macrophytes was enforced for present studies in Ansupa Lake, the largest freshwater lake of the state Odisha (India). Regular field inspection, quadratic sampling, and specimen collections were carried out to identify the present macrophytes of the lake and their quantitative aspects like frequency of occurrences, abundance, values of diversity indices, adaptation and growth forms, species distribution, etc. 244 macrophyte species were identified, including 182 semi-aquatic and 62 obligatory aquatic macrophytes. The latter group had 35\% submerged, 15\% free-floating, 31\% rooted floating, and 19\% marshy plant species. The comparison of growth form showed $66 \%$ annuals and the remaining $34 \%$ perennial plants. The diversity indices resulted, Simpson, complement index-0.561, Shannon-Weiner index-1.367, Species richness index 3.079, and Species evenness index-0.156. The study showed that the lake provides suitable habitats for the existence of a diverse group of macrophytes. Still, the extensive invasion of a few species has threatened the lake, which needs to be appropriately managed to restore the health of this natural resource for the benefit of humanity.
\end{abstract}

Keywords: Ansupa Lake, conservation, macrophyte diversity, species invasion

\section{INTRODUCTION}

Wetlands are the hotspots of biological diversity and invaluable for sustainable living. Plants in water are called macrophytes (Dodds 2002). They act as "biological engineers" restoring water quality (Byers et al. 2006). It includes flowering and non-flowering plants that start their lives in and around water bodies (Chambers et al., 2008). Some 2,614 aquatic vascular macrophytes occur globally, representing only $1 \%$ of the total number of vascular plants (Ansari et al., 2017). The total number of aquatic plant species in Indian freshwaters exceeds 1,200 (Gopal 1995). Many aquatic plant species are invasive species (Oyedeji and Abowei 2012). These plants cause local loss of species diversity and alter ecosystem structure, resulting in a significant negative impact on aquatic biodiversity and water quality (Brundu 2015; Chamier et al. 2012; Wang et al. 2016; Zedler and Zedler and Kercher 2004). In India, over 140 aquatic plants are reported to have attained the status of aquatic weeds in different situations (Gupta 2012; Naskar 1990; Shah and Reshi 2012; Varshney et al. 2008).

Ansupa Lake, the present study site, is the largest freshwater lake of the state Odisha (India) (Mohanty and Das 2008) and a lake of national importance (Das and Mohanty 2008). The lake provides livelihood provisions like fishing, i.e., small indigenous fishes, table-sized fishes, ornamental fishes; agriculture, i.e., rice cultivation; edible aquatic plants, and ecotourism due to its unique biodiversity and natural scenery (Sarkar et al. 2015). More than 25,000 fishermen and local residents live on the lake water (Das and Mohanty 2008; Mohanty and Das 2008). The average water depth of the lake was 4 meters (Das and Mohanty 2008). The lake receives annual rainfall between $800 \mathrm{~mm}$ to $1300 \mathrm{~mm}$ (Das and Mohanty 2008; Panda et al. 2016) and most during July and August each year. It hosts 44 species of phytoplankton, 32 species of zooplankton, and 30 species of fishes (Patra and Patra 2007). For the first time, Panda et al. (2016) reported the occurrence of Hygroryza aristata (Retz.) Nees. ex Wt. and Arn., a wild relative of edible rice in Ansupa Lake, as the only habitat in the state for this species. There are few published works on Ansupa Lake, and the macrophytes study is very poorly reported (Das and Mohanty 2008; Mohanty and Das 2008; Varshney et al. 2008; Sarkar et al. 2015; Panda et al. 2016). All previous studies reported the progressive degradation conditions of the lake due to siltation, shrinkage of water spread area, and invasions of aquatic plants (Das and Mohanty 2008; Mohanty and Das 2008; Sarkar et al. 2015; Panda et al. 2016).

Knowing the importance of Ansupa Lake, present studies were designed to identify the macrophyte diversity. These problematic weeds need to be appropriately managed to conserve indigenous biota and create better livelihood opportunities from the lake. 


\section{MATERIALS AND METHODS}

\section{Study area}

Ansupa Lake is the largest freshwater lake of Odisha State, India, situated between latitude $20^{\circ} 26^{\prime} 21^{\prime \prime}$ to $20^{\circ}$ $28^{\prime} 52^{\prime \prime} \mathrm{N}$ and $85^{\circ} 36^{\prime} 25^{\prime \prime}$ to $85^{\circ} 36^{\prime} 0^{\prime \prime} \mathrm{E}$ longitude on the riverbank of Mahanadi (Figure 1). The lake area is around 375 acres and 385 acres during the dry and rainy seasons, respectively (Mohanty and Das 2008).

\section{Field data collection and floristic study}

The floristic studies were carried out during November 2014 and extensive regular fieldwork from April to November 2017. The recorded macrophytes were identified with the help of available regional and international scientific literature (Calvert and Liessmann 2014; Campbell et al. 2010; Crow and Hellquist 2000; Das 2012; Gerber et al. 2004; Ghosh 2005; Gupta 2012; Haines 19211925; Naskar 1990). The scientific name and author citation were checked with, The plant list (http://www.theplantlist.org/) and International Plant Names Index (http://www.ipni.org/ipni/plantnamesearchpage.do). Quantitative status and ecological parameters were calculated from 25 fixed random plots, i.e., size1m $\times 1 \mathrm{~m}$ (Figure 1).

\section{Data analysis}

The quadratic parameters like Frequency and Abundance (Upadhyay et al. 2009), Whitford's index (A/F) (Whitford 1949), Species richness index (Margalef 1958), Simpson complement index (1-D $)$ from Simpson Dominance index (Simpson 1949), Shannon-Wiener index (Shannon and Wiener 1963) and Species evenness index (J) (Pielou 1975) were calculated as follows:

$$
\begin{aligned}
& \text { Frequecy }=\frac{\text { No. of plots in which a species occurs }}{\text { Total no of plots sampled }} \times 100 \\
& \text { Abundance }=\frac{\text { Total number of individuals of a species in all quadrates }}{\text { Number of quadrates in which the species occured }} \\
& \text { Species dispersion or Whitford's index }(\mathrm{A} / \mathrm{F})=\frac{\text { Abundance }}{\text { Frequency }} \\
& \text { Species richness Index }(R T)=\frac{S-1}{\ln N} \quad \text { as per Margalef }(1958)
\end{aligned}
$$

Where, $S$ is the total number of species in the community and $\mathrm{N}$ is the total number of individuals of all species of a community.
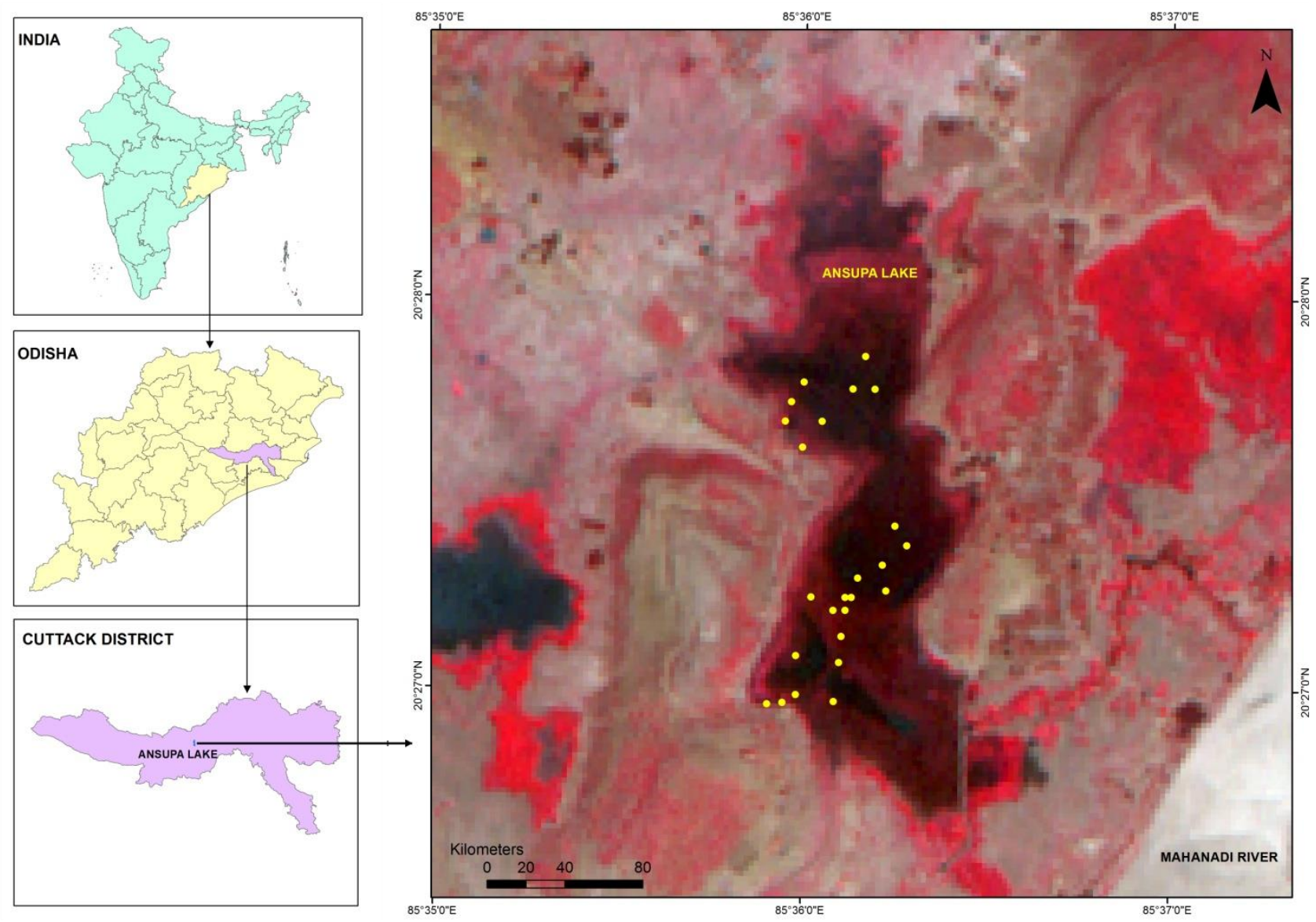

Figure 1. Location map of Ansupa Lake, Cuttack District, Odisha, India 
Simpson dominance index $\left(D_{5}\right)=\Sigma P i^{2}$ as per Simpson (1949)

Shannon - Wiener index $=-\sum \mathrm{Pi} \log _{\mathrm{n}} \mathrm{Pi}$ as per Shannon - Weiner $(1963)$

Where, $P i=\frac{\text { Number of individual of one species }}{\text { Total number of all individuals }}$

Species Evenness Index $(E I)=\frac{H^{\prime}}{\ln S}$ as per Pielou (1975)

Where, $\mathrm{H}^{\prime}$ is the Shannon-Weiner index of the community and $\mathrm{S}$ is the total number of species in the community.

\section{RESULTS AND DISCUSSION}

A total of 244 vascular macrophytes were identified to occur in shoreline areas of the lake. Out of the total record, 238 species were of flowering plants, i.e., Angiosperms (Table 1), and 6 species of non-flowering macrophytes, i.e., Pteridophyte (Table 2). All six pteridophytes were strictly aquatic species; they belong to only two families (i.e., Marsileaceae and Salviniaceae) except Azolla microphylla Kaulf, an annual species others were perennial in their growth form (Table 2). The angiospermic macrophytes belong to a total of sixty families. Poaceae and Cyperaceae were recorded as the most diversified families among these families (Figure 2). The classification of all the recorded macrophytes based on habitat preference showed 182 $(75 \%)$ semi-aquatic species and $62(25 \%)$ aquatic species (Figure 3). Categorization of total angiosperms revealed $137(58 \%)$ dicot species and $101(42 \%)$ monocot species (Figure 4). Among the dicot group, only 26 (19\%) species were strictly aquatic, and $111(81 \%)$ species were semiaquatic plants (Figure 5). Similarly, the monocot group had 30 species $(30 \%)$ and 71 species $(70 \%)$ as aquatic and semi-aquatic plants, respectively (Figure 6). The comparison of growth form showed 160 species (66\%) annual and the remaining 84 species $(34 \%)$ as perennial macrophytes (Figure 7). The classification of total aquatic species displayed 35\% submerged, $15 \%$ free-floating, 31\% rooted floating, and 19\% marshy plant species (Figure 8). The study of nativity resulted in 56 species out of 244 species as exotic or nonnative macrophytes of India (Table 1 and Table 2). The quadratic study revealed the quantitative status of 28 common macrophytes (Table 3 ). Maximum species diversity was recorded in the peripheral or shoreline plots. The most frequent and abundant species were Ceratophylum demersum L., Hydrila verticelastar (L.) Pers., Nelumbo nucefera Gaertn., Najas sp., Utricularia sp., Eichhornia crassipes (Mart.) Solm-Laub. and Salvinia molesta D. S. Mitch from the lake's interior. Other species like Polygonum barbatum L., Hymenachne amplexicaulis (Rudge) Nees, Cyperus iria L., Alternanthera philoxeroides A. St-Hil., Cyperus rotundus L. were more abundant at the land water interface (i.e., marshy areas). The distribution pattern (i.e., Whitford's index) showed all species with mthe ore or less contagious types of distribution $(\mathrm{A} / \mathrm{F}>0.05)$. The diversity indices study showed Simpson complement index-0.561, ShannonWeiner index-1.367, Species richness index 3.079, and Species evenness index-0.156 (Figure 9).

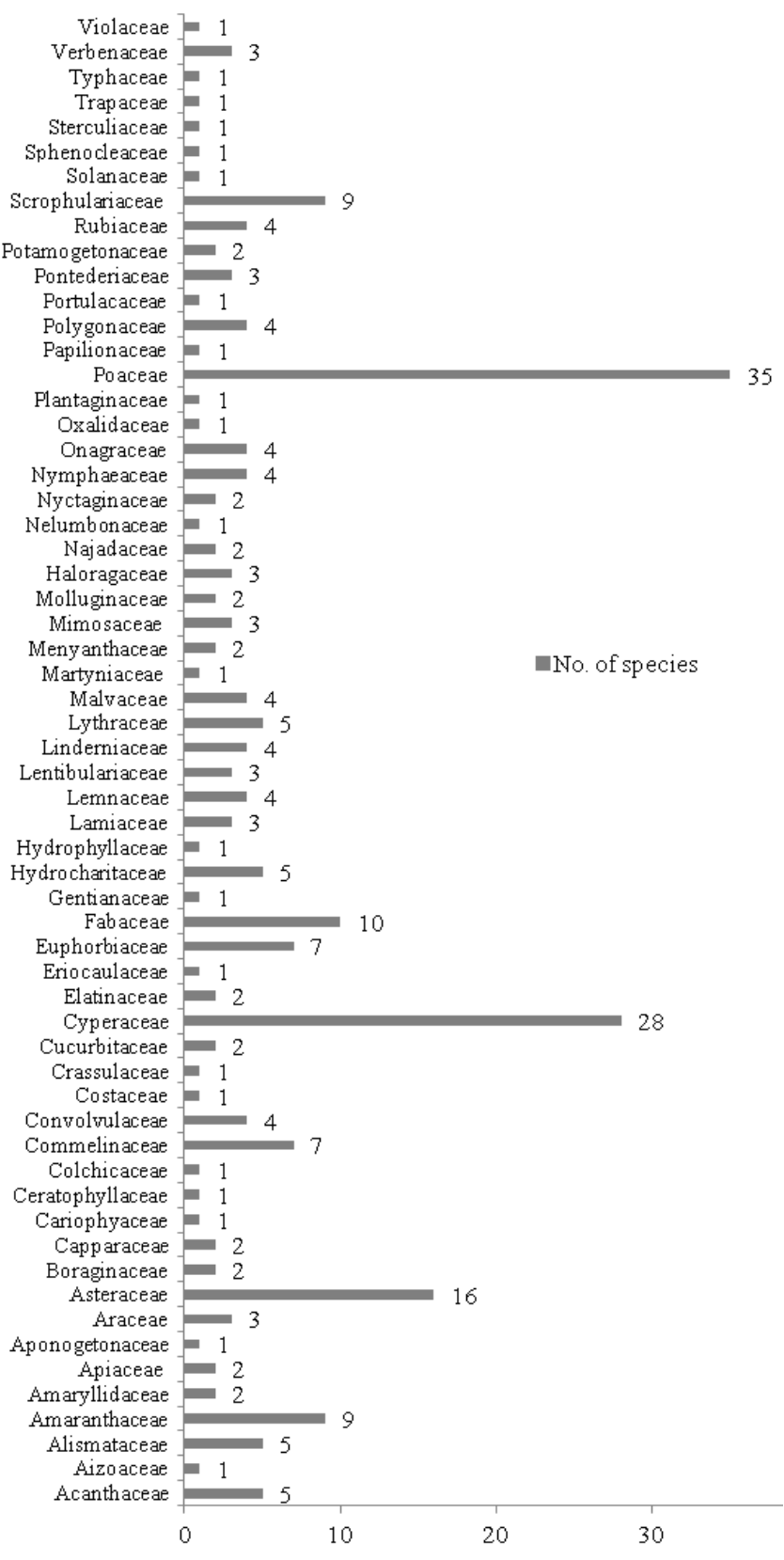

Figure 2. Family wise recorded number of angiospermic macrophytes species

The study found the occurrence of wide habitat variability that helped the establishment of a different group of aquatic and semi-aquatic vascular macrophytes in the lake. Many macrophytes showed seasonal changes of population status, influenced by water level (Dalu et al., 2012). This affects the value of the diversity index of the ecosystem, as calculated by the ratio between the number of species and the number of individuals in that community (Ansari et al., 2017). The low value of species evenness index showed the present species were not equally abundant; some species dominated over others. The lake hosts some unique macrophytes rarely found elsewhere in the state. Hygroryza aristata (Retz.) Nees. Ex Wt. \& Arn. and Oryza rufipogon Griff., the wild 


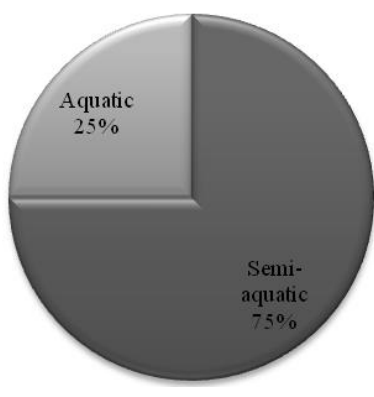

Figure 3. Classification as per habitat requirement: Aquatic and semi-aquatic plants (\%)

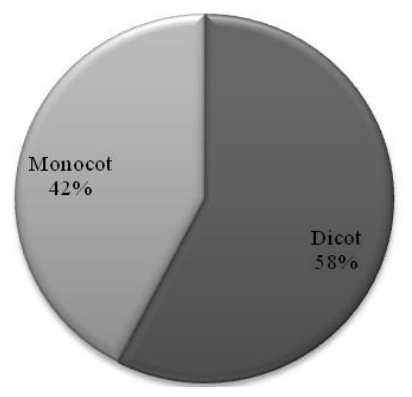

Figure 4. Classification into Angiosperm group: Diversity of dicot and monocot species (\%)

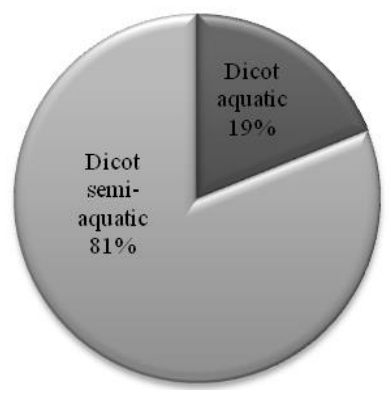

Figure 5. Classification of dicots into habitat group: Aquatic and semi-aquatic dicots $(\%)$

relative of edible rice was a common occurrence in the lake (Plate 1). The aesthetically essential and endangered plant species, Gloriosa superba L., has been recorded from shoreline areas of the lake for the first time (Plate 1). The semi-aquatic plants were diverse, and many showed seasonal growth. Many of them were small herbaceous annual plants.

Strong infestation of Nelumbo nucifera Gaertn., Eichhornia crassipes (Mart.) Solm-Laub., Salvinia molesta D. S. Mitch, Ceratophyllum demersum L., Hydrilla verticillata (L.f.) Royle, Najas indica (Willd) Cham.; Hymenachne amplexicaulis (Rudge) Nees, other grasses, and marshy vegetation were found negatively affecting the lake (Plate 2). Soil erosion from surrounding hills and siltation decreased water flow due to the closing of inlets and outlets with Mahanadi River; intensive fertilizer load is the possible factors for degradation of the lake.

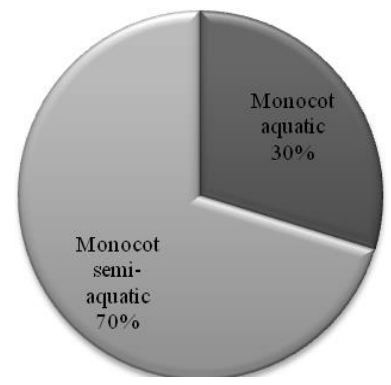

Figure 6. Classification of monocots into habitat groups: Aquatic and semi-aquatic monocots (\%)

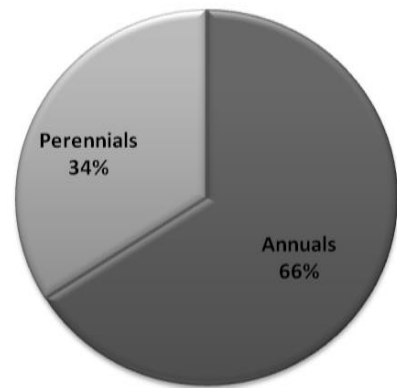

Figure 7. Classification of macrophytes into growth forms: Growth form of macrophytes (\%)

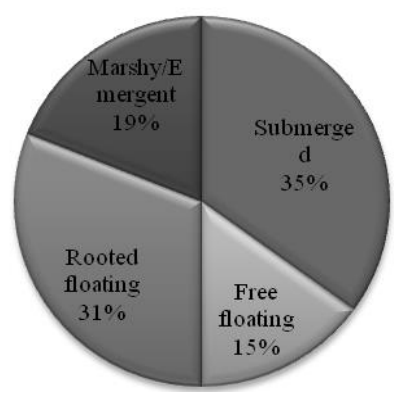

Figure 8. Classification of aquatic plants into their adaptation group: Adaptation forms of aquatic plants (\%)

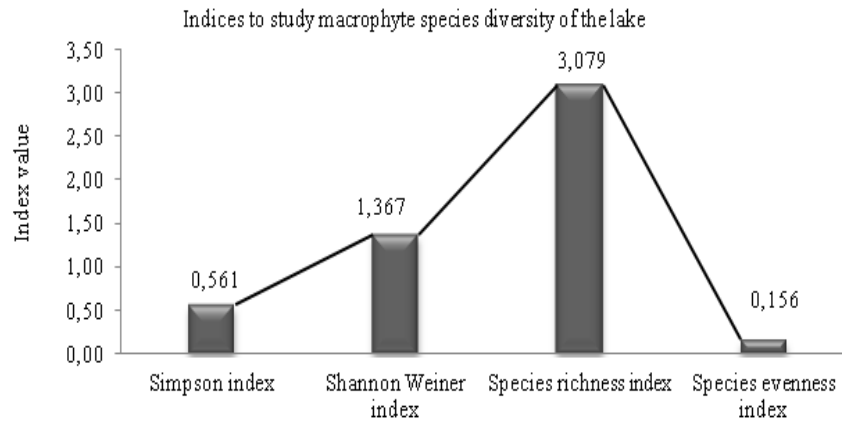

Figure 9. Diversity indices from quadrate data 
Table 1. List of Angiospermic macrophyte recorded from Ansupa Lake, Odisha, India

\begin{tabular}{|c|c|c|c|c|c|}
\hline Plant family & Si. No. & Plant species & $\begin{array}{l}\text { Plant } \\
\text { group }\end{array}$ & $\begin{array}{c}\text { Macrophyte } \\
\text { type }\end{array}$ & $\begin{array}{c}\text { Life } \\
\text { form }\end{array}$ \\
\hline \multirow[t]{5}{*}{ Acanthaceae } & 1 & Andrographis paniculata (Burm.f.) Wall. ex Nees & $\mathrm{D}$ & Semi-aquatic & Annual \\
\hline & 2 & Hygrophila auriculata (Schum) Heine & $\mathrm{D}$ & Semi-aquatic & Annual \\
\hline & 3 & Hygrophila schulli (Buch.-Ham.) M.R.Almeida \& S.M. Almeida & $\mathrm{D}$ & Semi-aquatic & Annual \\
\hline & 4 & Justicia diffusa Willd & $\mathrm{D}$ & Semi-aquatic & Annual \\
\hline & 5 & ${ }^{*}$ Ruellia tuberosa $\mathrm{L}$. & $\mathrm{D}$ & Semi-aquatic & Annual \\
\hline Aizoaceae & 6 & ${ }^{*}$ Trianthema portulacastrum $\mathrm{L}$. & $\mathrm{D}$ & Semi-aquatic & Annual \\
\hline \multirow[t]{5}{*}{ Alismataceae } & 7 & ${ }^{*}$ Alisma plantago-aquatica $\mathrm{L}$. & M & Aquatic (S) & Annual \\
\hline & 8 & Limnophyton obtusifolium (L.) Miq. & M & Aquatic (S) & Annual \\
\hline & 9 & Sagitaria sagittifolia L. & M & Aquatic (S) & Annual \\
\hline & 10 & Sagittaria guayanensis var. lappula D. Don & M & Aquatic (S) & Annual \\
\hline & 11 & Sagitaria trifolia L. & M & Aquatic (S) & Annual \\
\hline \multirow[t]{9}{*}{ Amaranthaceae } & 12 & ${ }^{*}$ Achyranthes aspera $\mathrm{L}$. & $\mathrm{D}$ & Semi-aquatic & Annual \\
\hline & 13 & Aerva lanata (L.) Juss. ex Schult. & $\mathrm{D}$ & Semi-aquatic & Annual \\
\hline & 14 & ${ }^{*}$ Alternanthera paronychioides A. St-Hil. & $\mathrm{D}$ & Semi-aquatic & Annual \\
\hline & 15 & ${ }^{*}$ Alternanthera philoxeroides (Mart.) Griseb. & $\mathrm{D}$ & Semi-aquatic & Annual \\
\hline & 16 & ${ }^{*}$ Alternanthera sessilis (L.) DC. & $\mathrm{D}$ & Semi-aquatic & Annual \\
\hline & 17 & *Amaranthus spinosus L. & $\mathrm{D}$ & Semi-aquatic & Annual \\
\hline & 18 & ${ }^{*}$ Amaranthus viridis $\mathrm{L}$. & $\mathrm{D}$ & Semi-aquatic & Annual \\
\hline & 19 & ${ }^{*}$ Celosia argentea $\mathrm{L}$. & $\mathrm{D}$ & Semi-aquatic & Annual \\
\hline & 20 & ${ }^{*}$ Gomphrena celosioides Mart. & $\mathrm{D}$ & Semi-aquatic & Annual \\
\hline \multirow{2}{*}{ Amaryllidaceae } & 21 & Crinum latifolium $\mathrm{L}$. & $\mathrm{M}$ & Aquatic (S) & Annual \\
\hline & 22 & Crinum viviparum (Lam.) R.Ansari \& V.J.Nair & $\mathrm{M}$ & Aquatic (RF) & Annual \\
\hline \multirow[t]{2}{*}{ Apiaceae } & 23 & Centella asiatica $(\mathrm{L}$.$) Urb.$ & $\mathrm{D}$ & Semi-aquatic & Perennial \\
\hline & 24 & ${ }^{*}$ Hydrocotyle modesta Cham. \& Schltdl. & $\mathrm{D}$ & Semi-aquatic & Perennial \\
\hline \multirow[t]{2}{*}{ Aponogetonaceae } & 25 & Aponogeton natans (L.) Engl. \& Krause & M & Aquatic (S) & Annual \\
\hline & 26 & Alocasia indica (Roxb.) Schott & M & Semi-aquatic & Perennial \\
\hline \multirow[t]{2}{*}{ Araceae } & 27 & Colocasia esculenta (L.) Schott & M & Semi-aquatic & Perennial \\
\hline & 28 & ${ }^{*}$ Pistia stratiotes $\mathrm{L}$. & M & Aquatic (FF) & Perennial \\
\hline \multirow[t]{16}{*}{ Asteraceae } & 29 & ${ }^{*}$ Ageratum conyzoides $\mathrm{L}$. & $\mathrm{D}$ & Semi-aquatic & Perennial \\
\hline & 30 & Blumea lacera (Burm.f.) DC. & $\mathrm{D}$ & Semi-aquatic & Annual \\
\hline & 31 & Caesulia axillaris Roxb. & $\mathrm{D}$ & Semi-aquatic & Annual \\
\hline & 32 & ${ }^{*}$ Chromolaena odorata (L.) King \& H.E. Robins. & $\mathrm{D}$ & Semi-aquatic & Perennial \\
\hline & 33 & Cyanthillium cinereum (L.) H. Rob & $\mathrm{D}$ & Semi-aquatic & Annual \\
\hline & 34 & ${ }^{*}$ Eclipta alba $(\mathrm{L})$. & $\mathrm{D}$ & Semi-aquatic & Annual \\
\hline & 35 & Eclipta prostrata (L.) L. & $\mathrm{D}$ & Semi-aquatic & Annual \\
\hline & 36 & Enydra fluctuans Lour. & $\mathrm{D}$ & Aquatic (S) & Annual \\
\hline & 37 & Emilia sonchifolia $($ L.) DC & $\mathrm{D}$ & Semi-aquatic & Annual \\
\hline & 38 & ${ }^{*}$ Gnaphalium polycaulon Pers. & $\mathrm{D}$ & Semi-aquatic & Annual \\
\hline & 39 & Grangea maderaspatana L. & $\mathrm{D}$ & Semi-aquatic & Annual \\
\hline & 40 & ${ }^{*}$ Mikania cordata (Burm.f.) Robinson & $\mathrm{D}$ & Semi-aquatic & Annual \\
\hline & 41 & Sphaeranthus indicus L. & $\mathrm{D}$ & Semi-aquatic & Annual \\
\hline & 42 & Spilanthes paniculata Wall. Ex DC. & $\mathrm{D}$ & Semi-aquatic & Annual \\
\hline & 43 & *Synedrella nodiflora (L.) Gaertn. & $\mathrm{D}$ & Semi-aquatic & Annual \\
\hline & 44 & ${ }^{*}$ Xanthium strumarium $\mathrm{L}$. & $\mathrm{D}$ & Semi-aquatic & Annual \\
\hline \multirow[t]{2}{*}{ Boraginaceae } & 45 & Coldenia procumbens $\mathrm{L}$. & $\mathrm{D}$ & Semi-aquatic & Annual \\
\hline & 46 & Heliotropium indicum $\mathrm{L}$. & $\mathrm{D}$ & Semi-aquatic & Annual \\
\hline \multirow[t]{2}{*}{ Capparaceae } & 47 & Cleome monophylla $\mathrm{L}$. & $\mathrm{D}$ & Semi-aquatic & Annual \\
\hline & 48 & Cleome viscosa $\mathrm{L}$. & $\mathrm{D}$ & Semi-aquatic & Annual \\
\hline Cariophyaceae & 49 & ${ }^{*}$ Polycarpon prostratum (Forssk.) Asc. \& Sch. & $\mathrm{D}$ & Semi-aquatic & Annual \\
\hline Ceratophyllaceae & 50 & Ceratophyllum demersum L. & $\mathrm{D}$ & Aquatic (S) & Perennial \\
\hline Colchicaceae & 51 & Gloriosa superba L. & M & Semi-aquatic & Perennial \\
\hline \multirow[t]{7}{*}{ Commelinaceae } & 52 & Commelina benghalensis $\mathrm{L}$. & M & Semi-aquatic & Perennial \\
\hline & 53 & Commelina erecta $\mathrm{L}$. & M & Semi-aquatic & Perennial \\
\hline & 54 & Commelina longifolia Lam. & M & Semi-aquatic & Perennial \\
\hline & 55 & Cyanotis axillaris (L.) D.Don ex Sweet & M & Semi-aquatic & Perennial \\
\hline & 56 & ${ }^{*}$ Evolvulus nummularius (L.) L. & M & Semi-aquatic & Perennial \\
\hline & 57 & Murdannia nudiflora (Linn.) Brenan. & M & Semi-aquatic & Annual \\
\hline & 58 & Murdannia spirata (L.) Bruckn. & M & Semi-aquatic & Annual \\
\hline \multirow[t]{2}{*}{ Convolvulaceae } & 59 & ${ }^{*}$ Ipomoea aquatica Forssk. & $\mathrm{D}$ & Aquatic (RF) & Perennial \\
\hline & 60 & *Ipomoea carnea Jacq. ssp. Fistulosa (Mart. ex Choisy) Austin & $\mathrm{D}$ & Semi-aquatic & Perennial \\
\hline
\end{tabular}




\begin{tabular}{|c|c|c|c|c|c|}
\hline & 61 & *Ipomoea pes-tigridis L. & $\mathrm{D}$ & Semi-aquatic & Perennial \\
\hline & 62 & Merremia tridentata (L.) Hall. f. & $\mathrm{D}$ & Semi-aquatic & Perennial \\
\hline Costaceae & 63 & Costus speciosus (J.Koenig) Sm. & M & Semi-aquatic & Perennial \\
\hline Crassulaceae & 64 & Bryophyllum calycinum Salisb. & $\mathrm{D}$ & Semi-aquatic & Perennial \\
\hline \multirow[t]{2}{*}{ Cucurbitaceae } & 65 & Mukia maderaspatana (L.) M. Roem. & $\mathrm{D}$ & Semi-aquatic & Annual \\
\hline & 66 & Cucumis melo $\mathrm{L}$. & $\mathrm{D}$ & Semi-aquatic & Annual \\
\hline \multirow[t]{28}{*}{ Cyperaceae } & 67 & Cyperus alopecuroides Rottb. & M & Semi-aquatic & Annual \\
\hline & 68 & ${ }^{*}$ Cyperus brevifolius (Rottb.) Hassk. & M & Semi-aquatic & Perennial \\
\hline & 69 & Cyperus cephalotes Vahl & M & Semi-aquatic & Perennial \\
\hline & 70 & Cyperus compressus $\mathrm{L}$. & M & Semi-aquatic & Annual \\
\hline & 71 & Cyperus corymbosus Rottb. & M & Semi-aquatic & Perennial \\
\hline & 72 & Cyperus difformis $\mathrm{L}$. & M & Semi-aquatic & Annual \\
\hline & 73 & Cyperus haspan $\mathrm{L}$ & M & Semi-aquatic & Annual \\
\hline & 74 & Cyperus imbricatus Retz. & M & Semi-aquatic & Perennial \\
\hline & 75 & Cyperus iria $\mathrm{L}$. & M & Semi-aquatic & Annual \\
\hline & 76 & Cyperus platystylis $\mathrm{R}$. Br. & M & Semi-aquatic & Perennial \\
\hline & 77 & Cyperus polystachyos Rottb. & M & Semi-aquatic & Perennial \\
\hline & 78 & Cyperus rotundus $\mathrm{L}$. & M & Semi-aquatic & Perennial \\
\hline & 79 & ${ }^{*}$ Cyperus strigosus L. & M & Semi-aquatic & Perennial \\
\hline & 80 & Eleocharis acutangula (Roxb.) schutt. & M & Aquatic (RE) & Perennial \\
\hline & 81 & Echinochloa crus-galli (L.) P. Beauv. & M & Semi-aquatic & Annual \\
\hline & 82 & Eleocharis dulcis (Burm.f.) Trin. ex Henschel & M & Semi-aquatic & Perennial \\
\hline & 83 & Fimbristylis dipsacea (Rottb.) C.B. Clarke & M & Semi-aquatic & Annual \\
\hline & 84 & Fimbristylis ferruginea (L) Vahl. & M & Semi-aquatic & Perennial \\
\hline & 85 & Fimbristylis littoralis Gaudich. & M & Semi-aquatic & Annual \\
\hline & 86 & Fimbristylis miliacea (L.) Vahl & M & Semi-aquatic & Annual \\
\hline & 87 & Fuirena ciliaris (L.) Roxb. & M & Semi-aquatic & Annual \\
\hline & 88 & ${ }^{*}$ Kyllinga tenuifolia Steud. & M & Semi-aquatic & Annual \\
\hline & 89 & Lipocarpha chinensis (Osbeck) J.Kern. & M & Semi-aquatic & Annual \\
\hline & 90 & Cyperous compactus Retz. & M & Semi-aquatic & Annual \\
\hline & 91 & Pycreus pumilus (L.) Nees & M & Semi-aquatic & Annual \\
\hline & 92 & Schoenoplectus articulatus (L.) Palla & M & Semi-aquatic & Annual \\
\hline & 93 & Schoenoplectus grossus (L.f.) Palla & M & Semi-aquatic & Perennial \\
\hline & 94 & Schoenoplectiella supina (L.) Lye & M & Semi-aquatic & Annual \\
\hline \multirow[t]{2}{*}{ Elatinaceae } & 95 & ${ }^{*}$ Bergia ammannioides Roxb. ex Roth & $\mathrm{D}$ & Semi-aquatic & Annual \\
\hline & 96 & Bergia capensis $\mathrm{L}$. & $\mathrm{D}$ & Semi-aquatic & Perennial \\
\hline Eriocaulaceae & 97 & Eriocaulon quinquangulare $\mathrm{L}$. & M & Semi-aquatic & Perennial \\
\hline \multirow[t]{7}{*}{ Euphorbiaceae } & 98 & Acalypha indica $\mathrm{L}$. & $\mathrm{D}$ & Semi-aquatic & Annual \\
\hline & 99 & ${ }^{*}$ Croton bonplandianus (Baill.) Kuntze & $\mathrm{D}$ & Semi-aquatic & Annual \\
\hline & 100 & Euphorbia hirta L. & $\mathrm{D}$ & Semi-aquatic & Annual \\
\hline & 101 & ${ }^{*}$ Euphorbia prostrata Aiton. & $\mathrm{D}$ & Semi-aquatic & Annual \\
\hline & 102 & Jatropha gossypiifolia $\mathrm{L}$. & $\mathrm{D}$ & Semi-aquatic & Perennial \\
\hline & 103 & ${ }^{*}$ Phyllanthus tenellus Roxb. & $\mathrm{D}$ & Semi-aquatic & Perennial \\
\hline & 104 & ${ }^{*}$ Ricinus communis $\mathrm{L}$. & $\mathrm{D}$ & Semi-aquatic & Perennial \\
\hline \multirow[t]{10}{*}{ Fabaceae } & 105 & Aeschynomene aspera $\mathrm{L}$. & $\mathrm{D}$ & Semi-aquatic & Annual \\
\hline & 106 & Aeschynomene indica $\mathrm{L}$. & $\mathrm{D}$ & Semi-aquatic & Annual \\
\hline & 107 & Alysicarpus vaginalis (L.) DC. & $\mathrm{D}$ & Semi-aquatic & Annual \\
\hline & 108 & ${ }^{*}$ Cassia tora $\mathrm{L}$ & $\mathrm{D}$ & Semi-aquatic & Annual \\
\hline & 109 & ${ }^{*}$ Crotalaria pallida Aiton & $\mathrm{D}$ & Semi-aquatic & Perennial \\
\hline & 110 & Crotalaria quinquefolia $\mathrm{L}$. & $\mathrm{D}$ & Semi-aquatic & Perennial \\
\hline & 111 & Zornia diphylla (L.) Pers. & $\mathrm{D}$ & Semi-aquatic & Annual \\
\hline & 112 & Senna obtusifolia (L.) H.S.Irwin. \& Barneby & $\mathrm{D}$ & Semi-aquatic & Annual \\
\hline & 113 & ${ }^{*}$ Senna occidentalis (L.) Link & $\mathrm{D}$ & Semi-aquatic & Annual \\
\hline & 114 & Sesbania bispinosa (Jacq.) W.F. Wt. & $\mathrm{D}$ & Semi-aquatic & Annual \\
\hline Gentianaceae & 115 & Hoppea dichotoma Willd. & $\mathrm{D}$ & Semi-aquatic & Annual \\
\hline \multirow{5}{*}{ Hydrocharitaceae } & 116 & Blyxa echinosperma (Clarke) Hook.f. & M & Aquatic (S) & Annual \\
\hline & 117 & Hydrilla verticillata (L.f.) Royle & M & Aquatic (S) & Perennial \\
\hline & 118 & Nechamandra alternifolia (Roxb. ex Wight) Thw. & M & Aquatic (S) & Perennial \\
\hline & 119 & Ottelia alismoides (L.) Pers. & M & Aquatic (S) & Perennial \\
\hline & 120 & Vallisneria natans (Lour.) H. Hara & M & Aquatic (S) & Annual \\
\hline Hydrophyllaceae & 121 & Hydrolea zeylanica (L.) Vahl. & $\mathrm{D}$ & Aquatic (RE) & Annual \\
\hline \multirow[t]{3}{*}{ Lamiaceae } & 122 & Anisomeles indica (L.) O. Kuntze. & $\mathrm{D}$ & Semi-aquatic & Perennial \\
\hline & 123 & Leucas aspera (Willd.) Link & $\mathrm{D}$ & Semi-aquatic & Annual \\
\hline & 124 & Pogostemon quadrifolius (Benth.) F. Muell. & $\mathrm{D}$ & Semi-aquatic & Annual \\
\hline \multirow[t]{2}{*}{ Lemnaceae } & 125 & ${ }^{*}$ Spirodela polyrrhiza (L.) Schleid. & M & Aquatic (FF) & Perennial \\
\hline & 126 & Lemna gibba $\mathrm{L}$. & M & Aquatic (FF) & Annual \\
\hline
\end{tabular}




\begin{tabular}{|c|c|c|c|c|c|}
\hline & 127 & Lemna aequinoctialis Welw & $\mathrm{M}$ & Aquatic (FF) & Annual \\
\hline & 128 & Wolffia globosa (Roxb.) Hartog \& Vander Plas & M & Aquatic (FF) & Annual \\
\hline \multirow[t]{3}{*}{ Lentibulariaceae } & 129 & Utricularia aurea Lour. & $\mathrm{D}$ & Aquatic (S) & Annual \\
\hline & 130 & Utricularia inflexa Forssk. & $\mathrm{D}$ & Aquatic (S) & Annual \\
\hline & 131 & Utricularia bifida $\mathrm{L}$. & $\mathrm{D}$ & Aquatic (S) & Annual \\
\hline Linderniaceae & 132 & Lindernia crustacea (L.) F.Muell. & $\mathrm{D}$ & Semi-aquatic & Annual \\
\hline \multirow[t]{5}{*}{ Lythraceae } & 133 & Ammannia baccifera $\mathrm{L}$. & $\mathrm{D}$ & Semi-aquatic & Annual \\
\hline & 134 & Ammannia multiflora Roxb. & $\mathrm{D}$ & Semi-aquatic & Annual \\
\hline & 135 & Ammannia octandra L.f. & $\mathrm{D}$ & Semi-aquatic & Annual \\
\hline & 136 & Rotala densiflora (Roth. ex Roem. \& Schult.) Koehne & $\mathrm{D}$ & Semi-aquatic & Annual \\
\hline & 137 & Rotala indica (Willd.) Koehne & $\mathrm{D}$ & Semi-aquatic & Annual \\
\hline \multirow[t]{4}{*}{ Malvaceae } & 138 & Abutilon indicum (L.) Sweet & $\mathrm{D}$ & Semi-aquatic & Annual \\
\hline & 139 & Corchorus aestuans L. & $\mathrm{D}$ & Semi-aquatic & Annual \\
\hline & 140 & Sida cordifolia $\mathrm{L}$. & $\mathrm{D}$ & Semi-aquatic & Annual \\
\hline & 141 & Urena lobata $\mathrm{L}$. & $\mathrm{D}$ & Semi-aquatic & Annual \\
\hline Martyniaceae & 142 & ${ }^{*}$ Martynia annua $\mathrm{L}$. & $\mathrm{D}$ & Semi-aquatic & Annual \\
\hline \multirow{2}{*}{ Menyanthaceae } & 143 & Nymphoides hydrophylla (Lour.) Kuntze & $\mathrm{D}$ & Aquatic (RF) & Annual \\
\hline & 144 & Nymphoides indica (L.) Kuntze & $\mathrm{D}$ & Aquatic (RF) & Annual \\
\hline \multirow[t]{3}{*}{ Mimosaceae } & 145 & ${ }^{*}$ Mimosa pudica L. & $\mathrm{D}$ & Semi-aquatic & Perennial \\
\hline & 146 & Neptunia oleracea Lour. & $\mathrm{D}$ & Aquatic (RF) & Perennial \\
\hline & 147 & Neptunia plena (L.) Benth. & $\mathrm{D}$ & Aquatic (RF) & Perennial \\
\hline \multirow[t]{2}{*}{ Molluginaceae } & 148 & Glinus oppositifolius (L.) Aug. DC & $\mathrm{D}$ & Semi-aquatic & Annual \\
\hline & 149 & Mollugo pentaphylla $\mathrm{L}$. & $\mathrm{D}$ & Semi-aquatic & Annual \\
\hline \multirow[t]{3}{*}{ Haloragaceae } & 150 & Myriophyllum tetrandrum Roxb. & $\mathrm{D}$ & Aquatic (RE) & Annual \\
\hline & 151 & "Myriophyllum aquaticum (Vell.) Verdc. & $\mathrm{D}$ & Aquatic (RE) & Perennial \\
\hline & 152 & Myriophyllum verticillatum $\mathrm{L}$. & $\mathrm{D}$ & Aquatic (RE) & Annual \\
\hline \multirow[t]{3}{*}{ Najadaceae } & 153 & Najas faveolata A. Br. ex Magam. & M & Aquatic (S) & Perennial \\
\hline & 154 & Najas indica (Willd) Cham. & M & Aquatic (S) & Perennial \\
\hline & 155 & Najas marina $\mathrm{L}$ & M & Aquatic (S) & Perennial \\
\hline Nelumbonaceae & 156 & Nelumbo nucifera Gaertn. & $\mathrm{D}$ & Aquatic (RF) & Perennial \\
\hline \multirow[t]{2}{*}{ Nyctaginaceae } & 157 & Boerhavia diffusa $\mathrm{L}$. & $\mathrm{D}$ & Semi-aquatic & Annual \\
\hline & 158 & Boerhavia repens $\mathrm{L}$. & $\mathrm{D}$ & Semi-aquatic & Annual \\
\hline \multirow{4}{*}{ Nymphaeaceae } & 159 & Euryale ferox Salisb. & $\mathrm{D}$ & Aquatic (RF) & Perennial \\
\hline & 160 & Nymphaea nouchali Burm.f. & $\mathrm{D}$ & Aquatic (RF) & Perennial \\
\hline & 161 & Nymphaea pubescens Willd. & $\mathrm{D}$ & Aquatic (RF) & Perennial \\
\hline & 162 & Nymphaea rubra Roxb. ex Andrews & $\mathrm{D}$ & Aquatic (RF) & Perennial \\
\hline \multirow[t]{4}{*}{ Onagraceae } & 163 & Ludwigia prostrata Roxb. & $\mathrm{D}$ & Semi-aquatic & Annual \\
\hline & 164 & Ludwigia adscendens (L.) H. Hara & $\mathrm{D}$ & Aquatic (RF) & Perennial \\
\hline & 165 & Ludwigia octovalvis (Jacq.) P.H. Raven & $\mathrm{D}$ & Semi-aquatic & Annual \\
\hline & 166 & Ludwigia perennis $\mathrm{L}$. & $\mathrm{D}$ & Semi-aquatic & Annual \\
\hline Oxalidaceae & 167 & Oxalis corniculata $\mathrm{L}$. & $\mathrm{D}$ & Semi-aquatic & Annual \\
\hline Plantaginaceae & 168 & ${ }^{*}$ Scoparia dulcis $\mathrm{L}$. & $\mathrm{D}$ & Semi-aquatic & Annual \\
\hline \multirow[t]{25}{*}{ Poaceae } & 169 & Apluda mutica $\mathrm{L}$. & M & Semi-aquatic & Annual \\
\hline & 170 & Arundinella pumila (Hochst. ex A.Rich) Steud & M & Semi-aquatic & Annual \\
\hline & 171 & Axonopus compressus (Sw.) P.Beauv. & M & Semi-aquatic & Perennial \\
\hline & 172 & Brachiaria deflexa (Schumach.) C.E.Hubb. ex Robyns & M & Semi-aquatic & Annual \\
\hline & 173 & Brachiaria mutica (Forssk.) Stapf. & M & Semi-aquatic & Perennial \\
\hline & 174 & Brachiaria ramosa (L.) Stapf & M & Semi-aquatic & Annual \\
\hline & 175 & Brachiaria reptans (L.) C.A.Gardner \& C.E.Hubb & M & Semi-aquatic & Annual \\
\hline & 176 & ${ }^{*}$ Chloris barbata $\mathrm{Sw}$ & M & Semi-aquatic & Annual \\
\hline & 177 & Cyrtococcum longipes (Hook.f.) A.Camus & M & Semi-aquatic & Perennial \\
\hline & 178 & Cynodon dactylon (L.) Pers. & M & Semi-aquatic & Perennial \\
\hline & 179 & *Dactyloctenium aegyptium (L.) Willd. & M & Semi-aquatic & Annual \\
\hline & 180 & Dichanthelium sp. & M & Semi-aquatic & Annual \\
\hline & 181 & Echinochloa colona (L.) Link & M & Semi-aquatic & Annual \\
\hline & 182 & Echinochloa crus-galli (L.) P.Beauv. & M & Semi-aquatic & Annual \\
\hline & 183 & Echinochloa stagnina (Retz.) Beauv. & M & Semi-aquatic & Annual \\
\hline & 184 & Eleusine indica (L.) Gaertn & M & Semi-aquatic & Annual \\
\hline & 185 & Elytrophorus spicatus (Willd.) A. Camus & M & Semi-aquatic & Annual \\
\hline & 186 & Eragrostis ciliaris $(\mathrm{L}$.$) R.Br.$ & M & Semi-aquatic & Annual \\
\hline & 187 & Eragrostis gangetica (Roxb.) Steudel & M & Semi-aquatic & Annual \\
\hline & 188 & Eragrostis japonica (Thunb.) Trin. & M & Semi-aquatic & Perennial \\
\hline & 189 & Eragrostis pilosa (L.) P.Beauv. & M & Semi-aquatic & Annual \\
\hline & 190 & Eragrostis tenella (L.) P.Beauv.ex Roem.\& Schult. & M & Semi-aquatic & Annual \\
\hline & 191 & Hygroryza aristata (Retz.) Nees ex Wight \& Arn & M & Aquatic (RF) & Perennial \\
\hline & 192 & ${ }^{*}$ Hymenachne amplexicaulis (Rudge) Nees & M & Aquatic (RF) & Perennial \\
\hline & 193 & Leersia hexandra Sw. & M & Semi-aquatic & Perennial \\
\hline
\end{tabular}




\begin{tabular}{|c|c|c|c|c|c|}
\hline & 194 & Oryza rufipogon Griff. & $\mathrm{M}$ & Semi-aquatic & Perennial \\
\hline & 195 & Panicum sumatrense Roth & M & Semi-aquatic & Perennial \\
\hline & 196 & ${ }^{*}$ Paspalum dilatatum Poir & M & Semi-aquatic & Annual \\
\hline & 197 & Paspalum distichum $\mathrm{L}$. & M & Semi-aquatic & Perennial \\
\hline & 198 & Paspalum vaginatum $\mathrm{Sw}$. & M & Semi-aquatic & Annual \\
\hline & 199 & Setaria pumila (Poir.) Roem. \& Schult. & M & Semi-aquatic & Annual \\
\hline & 200 & Saccharum spontaneum $\mathrm{L}$ & M & Semi-aquatic & Perennial \\
\hline & 201 & Setaria glauca (L.) Beauv. & M & Semi-aquatic & Annual \\
\hline & 202 & Sporobolus coromandelianus (Retzi.) Kunth & M & Semi-aquatic & Annual \\
\hline Papilionaceae & 203 & Sesbania bispinosa (Jacq.) W.Wight. & $\mathrm{D}$ & Semi-aquatic & Annual \\
\hline \multirow[t]{4}{*}{ Polygonaceae } & 204 & *Persicaria glabrum (Willd.) M.Gomez & $\mathrm{D}$ & Semi-aquatic & Perennial \\
\hline & 205 & ${ }^{*}$ Polygonum barbatum $\mathrm{L}$. & $\mathrm{D}$ & Semi-aquatic & Perennial \\
\hline & 206 & Polygonum plebeium $\mathrm{R}$. Br. & $\mathrm{D}$ & Semi-aquatic & Annual \\
\hline & 207 & *Rumex maritimus $\mathrm{L}$. & $\mathrm{D}$ & Semi-aquatic & Annual \\
\hline \multirow[t]{3}{*}{ Pontederiaceae } & 208 & Eichhornia crassipes (Mart.) Solm-Laub. & M & Aquatic (RF) & Perennial \\
\hline & 209 & Monochoria hastata (L.) Solm. & M & Aquatic (RF) & Perennial \\
\hline & 210 & Monochoria vaginalis (Burm f.) Presl. & M & Aquatic (RE) & Perennial \\
\hline Portulacaceae & 211 & Portulaca oleracea L. & $\mathrm{D}$ & Semi-aquatic & Annual \\
\hline \multirow[t]{2}{*}{ Potamogetonaceae } & 212 & *Potamogeton nodosus Poir. & M & Aquatic (S) & Annual \\
\hline & 213 & Stuckenia pectinata (L.) Börner & M & Aquatic (S) & Perennial \\
\hline \multirow[t]{4}{*}{ Rubiaceae } & 214 & Dentella repens (L.) Forst. et Forst. & $\mathrm{D}$ & Semi-aquatic & Annual \\
\hline & 215 & Oldenlandia diffusa (Willd.) Roxb. & $\mathrm{D}$ & Semi-aquatic & Annual \\
\hline & 216 & Mitracarpus hirtus (L.) DC. & $\mathrm{D}$ & Semi-aquatic & Annual \\
\hline & 217 & Oldenlandia corymbosa $\mathrm{L}$. & $\mathrm{D}$ & Semi-aquatic & Annual \\
\hline \multirow[t]{12}{*}{ Scrophulariaceae } & 218 & Bacopa monnieri (L.) Pennell. & $\mathrm{D}$ & Semi-aquatic & Annual \\
\hline & 219 & Dopatrium junceum (Roxb.) Buch-Ham. ex Benth. & $\mathrm{D}$ & Aquatic (RE) & Annual \\
\hline & 220 & Limnophila aquatica (Roxb.) Alston & $\mathrm{D}$ & Aquatic (RE) & Annual \\
\hline & 221 & Limnophila heterophylla (Roxb.) Benth. & $\mathrm{D}$ & Aquatic (RE) & Annual \\
\hline & 222 & Limnophila indica (L.) Druce & $\mathrm{D}$ & Aquatic (RE) & Annual \\
\hline & 223 & Limnophila sessiliflora (Vahl) Blume & $\mathrm{D}$ & Aquatic (RE) & Annual \\
\hline & 224 & Lindernia anagallis (Burm.f.) Pennel & $\mathrm{D}$ & Semi-aquatic & Annual \\
\hline & 225 & Lindernia antipoda (L.) Alston & $\mathrm{D}$ & Semi-aquatic & Annual \\
\hline & 226 & Lindernia parviflora (Roxb.) Haines & $\mathrm{D}$ & Semi-aquatic & Annual \\
\hline & 227 & Mecardonia procumbens (Mills.) Small & $\mathrm{D}$ & Semi-aquatic & Annual \\
\hline & 228 & Scoparia dulcis L. & $\mathrm{D}$ & Semi-aquatic & Annual \\
\hline & 229 & ${ }^{*}$ Verbascum chinense (L.) Santapau & $\mathrm{D}$ & Semi-aquatic & Annual \\
\hline Solanaceae & 230 & Physalis minima $\mathrm{L}$. & $\mathrm{D}$ & Semi-aquatic & Annual \\
\hline Sphenocleaceae & 231 & Sphenoclea zeylanica Gaertn. & $\mathrm{D}$ & Semi-aquatic & Annual \\
\hline Sterculiaceae & 232 & Melochia corchorifolia $\mathrm{L}$. & $\mathrm{D}$ & Semi-aquatic & Annual \\
\hline Trapaceae & 233 & Trapa natans L. var. bispinosa (Roxb.) Makino & $\mathrm{D}$ & Aquatic (RF) & Perennial \\
\hline Typhaceae & 234 & ${ }^{*}$ Typha angustata Bory \& Chaub. & M & Aquatic (RE) & Perennial \\
\hline \multirow[t]{3}{*}{ Verbenaceae } & 235 & *Lantana camara $\mathrm{L}$ & $\mathrm{D}$ & Semi-aquatic & Perennial \\
\hline & 236 & ${ }^{*}$ Lippia javanica (Burm.f.) Spreng. & $\mathrm{D}$ & Semi-aquatic & Perennial \\
\hline & 237 & Phyla nodiflora (L.) Greene & $\mathrm{D}$ & Semi-aquatic & Annual \\
\hline Violaceae & 238 & Hybanthus enneaspermus (L.) F.Muell. & $\mathrm{D}$ & Semi-aquatic & Annual \\
\hline
\end{tabular}

Note: $\mathrm{D}=$ Dicot, $\mathrm{M}=$ Monocot, $\mathrm{S}=$ Submerged, $\mathrm{FF}=$ Free floating, $\mathrm{RF}=$ Rooted floating, $\mathrm{RE}=$ Rooted erect, ${ }^{*}=$ Exotic or nonnative species (Un-marked species are native or indigenous to India)

Table 2. List of Non-flowering (Pteridophyte) macrophytes of Ansupa Lake (Odisha), India

\begin{tabular}{lllll}
\hline Family & S. No. & Plant species & Habitat group & Life form \\
\hline Marsileaceae & 1 & Marsilea minuta L. & Aquatic (RF) & Perennial \\
& 2 & Marsilea quadrifolia L. & Aquatic (RF) & Perennial \\
Salviniaceae & 3 & ${ }^{*}$ Azolla microphylla Kaulf. & Aquatic (FF) & Annual \\
& 4 & Azolla pinnata R.Br. & Aquatic (FF) & Perennial \\
& 5 & ${ }^{*}$ Salvinia minima Baker & Aquatic (FF) & Perennial \\
& 6 & ${ }^{*}$ Salvinia molesta D.S. Mitch & Aquatic (FF) & Perennial \\
\hline
\end{tabular}


Table 3. Quantitative status of important macrophytes of Ansupa Lake, Odisha, India

\begin{tabular}{|c|c|c|c|c|c|}
\hline Macrophyte species & $\begin{array}{l}\text { Total } \\
\text { count }\end{array}$ & $\begin{array}{c}\text { Total plots } \\
\text { where recorded }\end{array}$ & Frequency & Abundance & $\begin{array}{c}\begin{array}{c}\text { Abundance/ } \\
\text { frequency } \\
(\mathrm{A} / \mathbf{F})\end{array} \\
\end{array}$ \\
\hline Eichhornia crassipes (Mart.) Solm-Laub. & 31 & 4 & 16 & 7.75 & 0.484 \\
\hline Ipomoea aquatica Forssk. & 17 & 3 & 12 & 5.67 & 0.472 \\
\hline Cyperus strigosus L. & 14 & 2 & 8 & 7.0 & 0.875 \\
\hline Cyperus iria $\mathrm{L}$. & 60 & 1 & 4 & 60.0 & 15.00 \\
\hline Cyperus rotundus $\mathrm{L}$. & 20 & 1 & 4 & 20.0 & 5.00 \\
\hline Ludwigia adscendens (L.) H. Hara & 13 & 2 & 8 & 6.5 & 0.813 \\
\hline Ludwigia perennis $\mathrm{L}$. & 20 & 3 & 12 & 6.67 & 0.556 \\
\hline Alternanthera philoxeroides (Mart.) Griseb. & 25 & 1 & 4 & 25.0 & 6.250 \\
\hline Salvinia molesta D.S. Mitch & 37 & 3 & 12 & 12.33 & 1.028 \\
\hline Salvinia minima Baker & 6 & 1 & 4 & 6.0 & 1.500 \\
\hline Cyperus compressus $\mathrm{L}$. & 62 & 2 & 8 & 31.0 & 3.875 \\
\hline Kylling a tenuifolia Steud. & 2 & 1 & 4 & 2.0 & 0.500 \\
\hline Hydrilla verticillata (L.f.) Royle & 1240 & 12 & 48 & 103.33 & 2.153 \\
\hline Ceratophyllum demersum $\mathrm{L}$. & 4060 & 21 & 84 & 193.33 & 2.302 \\
\hline Najas faveolata A. Br. ex Magam. & 335 & 9 & 36 & 37.22 & 1.034 \\
\hline Nymphaea pubescens Willd. & 6 & 4 & 16 & 1.5 & 0.094 \\
\hline Trapa natans L. var. bispinosa (Roxb.) Makino & 8 & 1 & 4 & 8.0 & 2.00 \\
\hline Nelumbo nucifera Gaertn. & 57 & 16 & 64 & 3.56 & 0.056 \\
\hline Pistia stratiotes L. & 11 & 3 & 12 & 3.67 & 0.306 \\
\hline Spirodela polyrrhiza (L.) Schleid. & 54 & 4 & 16 & 13.5 & 0.844 \\
\hline Utricularia sp. & 171 & 4 & 16 & 42.75 & 2.672 \\
\hline Lemna gibba L. & 78 & 7 & 28 & 11.14 & 0.398 \\
\hline Azolla pinnata $\mathrm{R} \mathrm{Br}$. & 29 & 5 & 20 & 5.8 & 0.290 \\
\hline Polygonum barbatum L. & 38 & 1 & 4 & 38.0 & 9.500 \\
\hline Marsilea quadrifolia L. & 20 & 3 & 12 & 6.67 & 0.556 \\
\hline Aponogeton natans (L.) Engl. \& Krause & 5 & 1 & 4 & 5.0 & 1.250 \\
\hline Hygroryza aristata (Retz.) Nees ex Wight \& Arn & 7 & 2 & 8 & 3.5 & 0.438 \\
\hline Lindernia parviflora (Roxb.) Haines & 10 & 2 & 8 & 5.0 & 0.625 \\
\hline
\end{tabular}
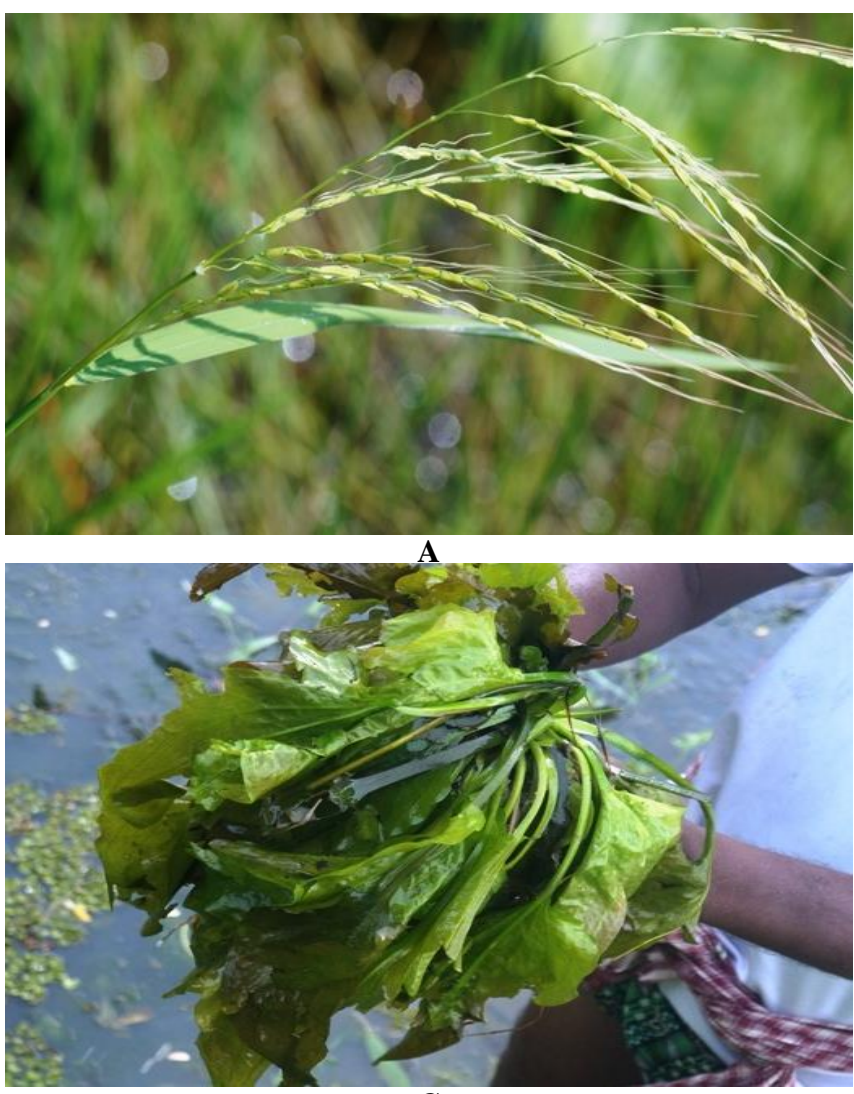

C
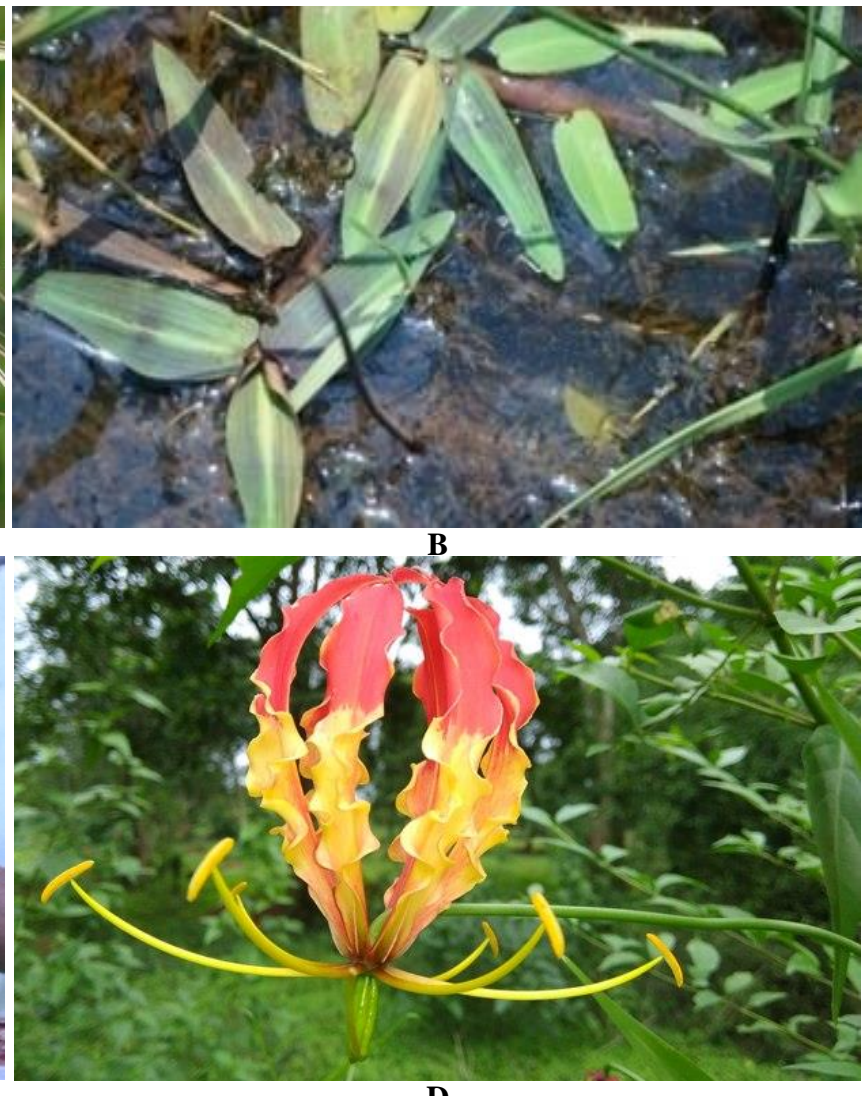

D

Plate 1. Some taxonomically important taxa from Ansupa Lake, Odisha, India. Note: A. Oryza rufipogon, B. Hygroryza aristata, C. Ottelia alismoides, D. Gloriosa superba 

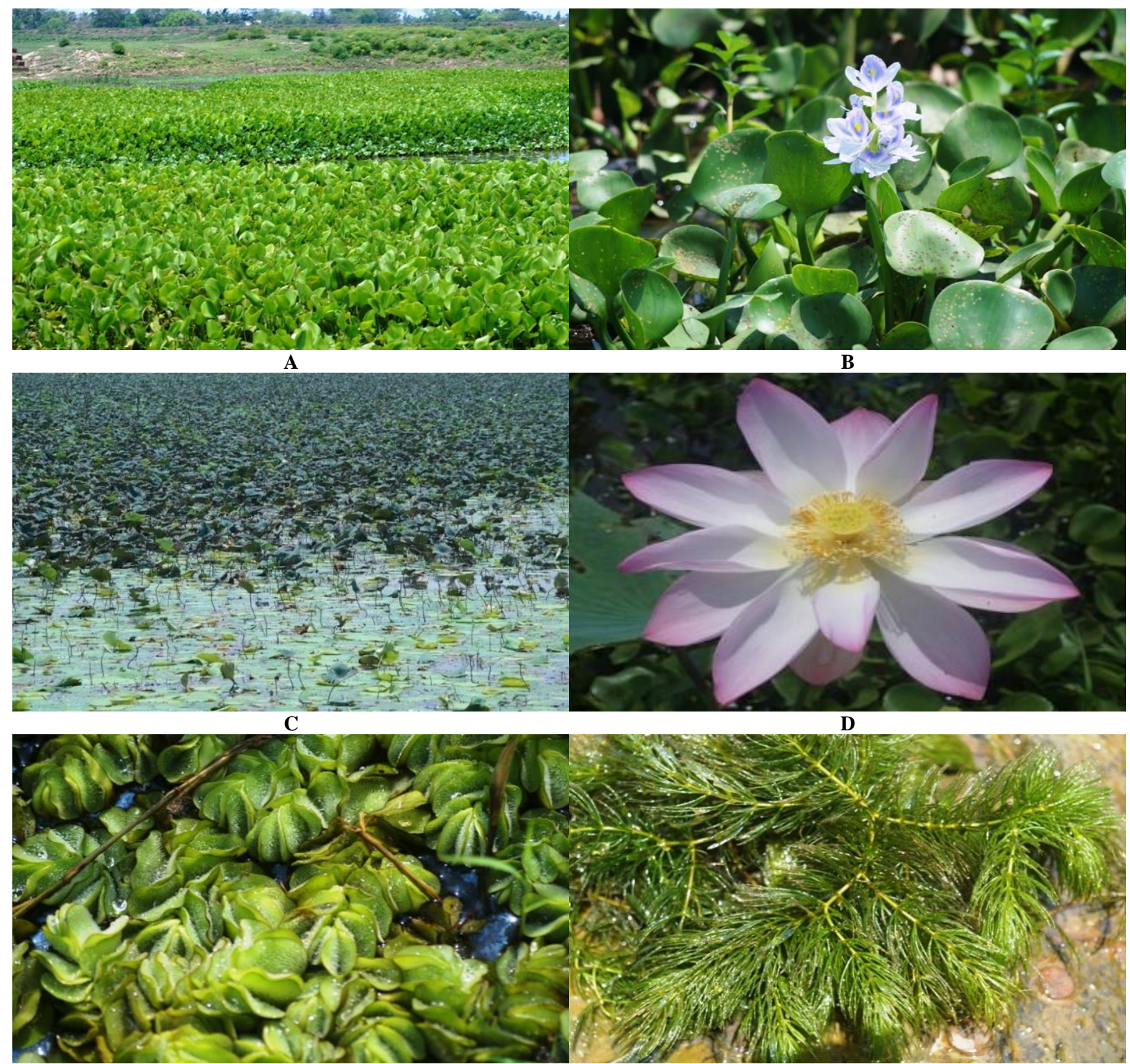

E

F

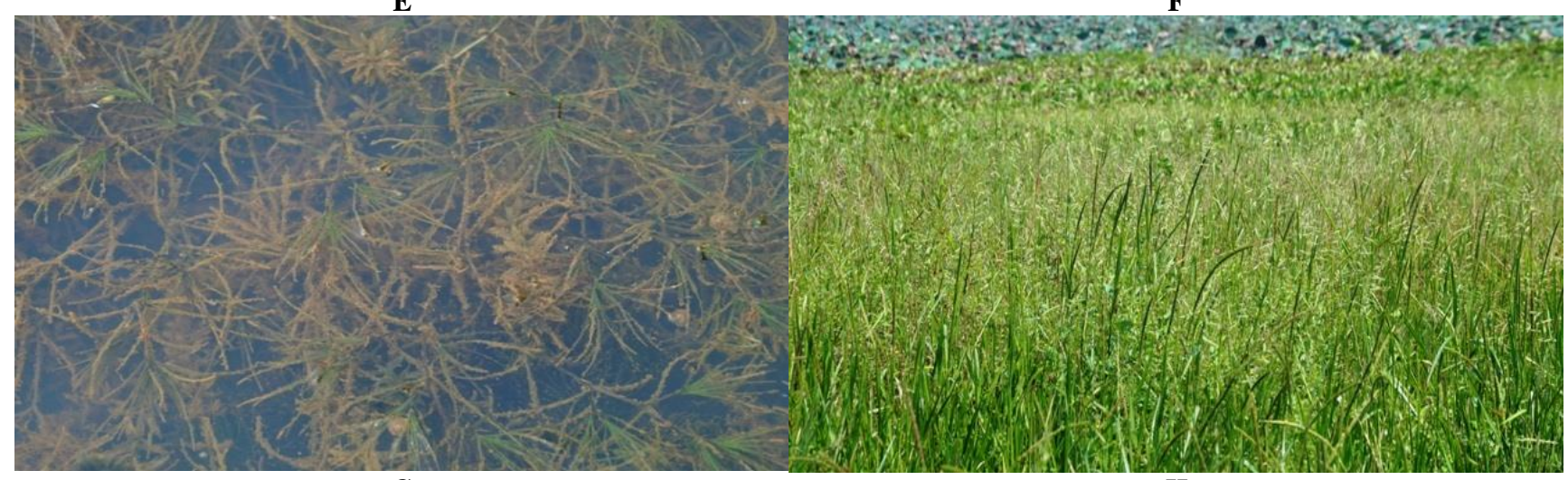

G

$\mathbf{H}$

Plate 2. Invasive weed species of Ansupa Lake, Odisha, India. Note: A-B. Eichhornia crassipes, C-D. Nelumbo nucifera, E. Salvinia molesta, F. Ceratophyllum demersum, G. Najas indica, H. Hymenachne amplexicaulis 
Besides having these troublesome weeds, the lake also hosts many macrophytes used as food, fodder, or medicine by the local households. Control of invasion and their management is tedious and needs multiple strategies. Management of this invasive grass must include a combination of techniques such as winter burning, herbicide application, and hydroperiod control. The floating rotted macrophyte Euryale ferox Salisb. once occurred in the lake (recorded in October 2014) is now extinct from the lake. Implementation of physical (mechanical) methods and dredging to required depth will reduce currently infested weeds and further regular monitoring, participation of both Governments agency and local community thought to restore a long-term functioning of the lake.

\section{General comments}

Aquatic macrophytes are an indispensable constituent of any wetland. They provide habitat to various aquatic fauna, act as primary producers, oxygenate the water, maintain water quality, do nutrient cycling, stabilize shoreline of lakes, provide substrate for growth of algae, provide shelter to benthic fauna and breeding ground for fishes, check the inflow of silt, reduce the nutrient load by self-utilizing, and minimize the development of algal blooms (Naskar 1990; Bornette and Puijalon 2009; Ansari et al. 2017). But, sometimes environments enforce and help invade exotic weeds in aquatic ecosystems, which negatively affect the entire ecosystem. These plants compete with native species and often facilitate the loss or extinction of less aggressive and indigenous species (Stallings et al., 2015). In many instances, they negatively affect human activities (e.g., fishing, swimming, navigation, and irrigation) and degrade the physical, chemical or biological aspects (Basak et al. 2015). In India, about 140 aquatic plants have been reported to have attained the status of aquatic weeds (Naskar 1990, Gupta 2012), many of them found in Ansupa Lake. The wetlands in India are also gradually shrinking and under severe anthropogenic pressure (Pattanaik et al., 2008; Udayakumar and Ajithadoss 2010). Regular physical visits, application of geospatial remote sensing techniques, monitoring change in floristic composition, maintaining required depth, reducing fertilizer use in agriculture in nearby cultivation lands, and creating green coverage in surrounding barren lands can save native biota from alien species to invade many aquatic ecosystems.

\section{ACKNOWLEDGEMENTS}

Authors are thanked full to the Ministry of Environment, Forest and Climate Change for providing financial assistance for Conservation and Management of Ansupa Lake, Odisha, India, for 2016-2017 under the National Plan for Conservation of Aquatic Eco-systems (NPCA).

\section{REFERENCES}

Ansari AA, Saggu S, Al-Ghanim SM, Abbas ZK, Gill SS, Khan FA, Dar MI, Naikoo MI, Khan AA. 2017. Aquatic plant biodiversity: A biological indicator for the monitoring and assessment of water quality. In: Ansari AA, Gill SS, Abbas ZK, Naeem M (eds) Plant Biodiversity: Monitoring, Assessment, and Conservation. CAB International, Wallingford. DOI: 10.1079/9781780646947.0218.

Basak SK, Ali MM, Islam MS, Shaha PR. 2015. Aquatic weeds of Haor area in Kishoregonj district, Bangladesh: Availability, threats, and management approaches. Intl J Fish Aquat Stud 2 (6): 151-156.

Bornette G, Puijalon S. 2009. Macrophytes: Ecology of Aquatic Plants. In Encyclopedia of Life Sciences (ELS). John Wiley \& Sons, Ltd., Chichester, UK. DOI: 10.1002/9780470015902.a0020475.

Brundu G. 2015. Plant invaders in European and Mediterranean inland waters: profiles, distribution, and threats. Hydrobiologia 746 (1): 61 79. DOI: 10.1007/s10750-014-1910-9.

Byers EJ, Cuddington K, Jones CG, Talley TS, Hastings A, Lambrinos JG, Crooks JA, Wilson WG. 2006. Using ecosystem engineers to restore ecological systems. Trends Ecol Evol 21 (9): 493-500. DOI: 10.1016/j.tree.2006.06.002

Calvert G, Liessmann L. 2014. Wetland Plants of the TownsvilleBurdekin Flood Plain. Lower Burdekin Landcare Association Inc., Ayr.

Campbell S, Higman P, Slaughter B, Schools E. 2010. A Field Guide to Invasive Plants of Aquatic and Wetland Habitats for Michigan. Michigan State University Extension, East Lansing, MI, USA.

Chambers PA, Lacoul P, Murphy KJ, Thomaz SM. 2008. Global diversity of aquatic macrophytes in freshwater. Hydrobiologia 595: 9-26. DOI: 10.1007/s10750-007-9154-6.

Chamier J, Schachtschneider K, Maitre DC, Ashton PJ, Wilgen BW. 2012. Impacts of invasive alien plants on water quality, with particular emphasis on South Africa. Water SA 38 (2): 345-356. DOI: 10.4314/wsa.v38i2.19.

Crow GE, Hellquist CB. 2000. Aquatic and Wetland Plants of Northeastern North America. The University of Wisconsin Press, Madison, WI

Dalu T, Clegg B, Nhiwatiwa T. 2012. Aquatic macrophytes in a tropical African reservoir: diversity, communities and the impact of reservoirlevel fluctuations. Trans R Soc S A 67 (3): 117-125. DOI: 10.1080/0035919X.2012.712554

Das CR, Mohanty S. 2008. Integrate Sustainable Environmental Conservation of Ansupa Lake: A famous water resource of Orissa, India. Water Energ Intl 65 (4): 62-66.

Das NR. 2012. Introduction to Aquatic and Semi-aquatic Plants of India. Kalyani Publishers, Punjab, India.

Dodds WK. 2002. Freshwater Ecology: Concepts and Environmental Applications. Academic Press, New York.

Gerber A, Cilliers CJ, Ginkel C. van, Glen R. 2004. Easy Identification of Aquatic Plants: A Guide for the Identification of Water Plants in and Around South African Impoundments. South Africa Department of Water Affairs, Pretoria.

Ghosh SK. 2005. Illustrated Aquatic and Wetland Plants in Harmony with Mankind. Standard Literature, 76, Acharya Jagadish Chandra Bose Road, Kolkata, India.

Gopal B. 1995. Biodiversity in Freshwater Ecosystems Including Wetlands, Biodiversity and Conservation in India, A Status Report. Zoological Survey of India, Calcutta, India.

Gupta OP. 2012. Weedy Aquatic Plants: Their Utility, Menace, and Management. Agrobios, India.

Haines HH. 1921-1925. The Botany of Bihar and Orisha, 6 parts. London, Botanical Survey of India, Culcutta.

Margalef DR. 1958. Information Theory in Ecology. Year Book of the Society for General Systems Research 3: 36-71.

Mohanty S, Das CR. 2008. Community Mobilization and Participation in Implementing Integrated Sustainable Conservation of Ansupa Lake, a Famous Wetland of Orissa. In: Sengupta M, Dalwani R (eds) Proceedings of Taal 2007: The $12^{\text {th }}$ World Lake Conference.

Naskar KR. 1990. Aquatic \& Semi-Aquatic Plants of the Lower Ganga Delta. Daya Publishing House, Delhi.

Oyedeji AA, Abowei JFN. 2012. The classification, distribution, control, and economic importance of aquatic plants. Intl J Fish Aquat Sci 1 (2): 118-128. 
Panda SP, Sahoo HK, Subudhi HN, Sahu AK, Mishra P. 2016. Ecofloristic diversity of Ansupa Lake, Odisha (India) with specia reference to aquatic macrophytes. J Biodiv Photon 116: 537-552.

Patra S, Patra AK. 2007. Environment impact assessment of a fresh water ecosystem operating in Ansupa Lake: An urgent need for development of fishery resources. Proc. Nat. Sem. on Environment pollution and its protection issues in Orissa. Organised by Department of Zoology G.S.College. Athagarh, 1-2 Sept. 95-98.

Pattanaik C, Prasad SN, Reddy CS. 2008. Warning bells in Ansupa Lake, Orissa. Curr Sci 94 (5): 560.

Pielou EC. 1975. Ecological Diversity. John Wiley and Sons, New York.

Sarkar SD, Ekka A, Sahoo AK, Rashith CM, Lianthuamluaia Roychowdhury A. 2015. Role of floodplain wetlands in supporting livelihood: A case study of Ansupa Lake in Odisha. J Environ Sci Comput Sci Eng Technol 4 (3): 819-826.

Shah MA, Reshi ZA. 2012. Invasion by alien macrophytes in freshwater ecosystems of India. In: Bhatt et al. (eds) Invasive Alien Plants: An Ecological Appraisal for the Indian Subcontinent. CAB International, Wallingford, UK. DOI: 10.1079/9781845939076.0199.

Shannon CE, Wiener W. 1963. The Mathematical Theory of Communication. University of Illinois Press, Urbana, USA

Simpson EH. 1949. Measurement of diversity. Nature 163: 688. DOI: $10.1038 / 163688 \mathrm{a} 0$
Stallings KD, Seth-Carley D, Richardson RJ. 2015. Management of Aquatic Vegetation in the Southeastern United states. J Integrat Pest Manag 6 (1): 1-5. DOI: 10.1093/jipm/pmv002.

Udayakumar M, Ajithadoss K. 2010. Angiosperms, Hydrophytes of five ephemeral lakes of Thiruvallur District, Tamil Nadu, India. Chicklist 6 (2): 270-274. DOI: 10.15560/6.2.270.

Upadhyay VP, Malviya HS, Behura S, Rout DK. 2009. Ecological methods for biodiversity assessment in EIA. Indian J Environ Ecoplan 16 (1): 157-168.

Varshney JG, Sushilkumar, Mishra JS. 2008. Current Status of Aquatic Weeds and Their Management in India. In: Sengupta M, Dalwani R (eds) Proceedings of Taal 2007: The 12th World Lake Conference. Jaipur, India, 28 October-2 November 2007.

Wang H, Wang Q, Bowler PA, Xiong W. 2016. Invasive aquatic plants in China. Aquat Invas 11 (1): 1-9. DOI: 10.3391/ai.2016.11.1.01.

Whitford PB. 1949. Distribution of woodland plants in relation to succession and clonal growth. Ecology 30 (2): 199-208. DOI: $10.2307 / 1931186$

Zedler JB, Kercher S. 2004. Causes and consequences of invasive plants in wetlands: opportunities, opportunists, and outcomes. Crit Rev Plant Sci 23 (5): 431-452. DOI: 10.1080/07352680490514673. 